\title{
Impact of Oppressors on Truthfulness: A Case Study on University of Karachi - Students (Under-Graduate \& Graduate)
}

A perspective which influence on prevalence and existence of truth within our own surroundings, keeping in contact with oppressors (barriers) and the four elements of revealing the truth (personalities).

\author{
Syed Shahid Zaheer Zaidi $\quad$ Muhammad Azhar Ali \\ Syed Shoeb Ahmed Farhat Hussain \\ Syed Yasir Zaheer Muhammad Kamran Khan
}

\begin{abstract}
Under this truth model and research study, the truth is defined as a central fold, which is communicated of its surroundings. Truth is always there available to every person for being revealed but each type of personality is distracted, distressed, dominated or oppressed with an oppressor who is a hurdle in prevalence of truth to each personality. It might be a voice, noise, a physical barrier or distortion etc.. The communication type between each and every elements of the model is extra personal in nature. The truth to any one type whether, through direct or indirect interaction. Therefore, the model responds for two-way communication process where, each entity or element act as sender and as well as receiver in the same discipline. The model and research study will further help in finding the core hypothesis i.e. to calculate when the ability of truthfulness in under-graduate, post-graduate and graduate standard students come into existence when dealing with various oppressive conditions and policies i.e. being unaware with the truth, and reacted when dealt with the situation of known about the truth, furthermore; what sources are acquired for prevalence of truth. Secondly, the inheritance element in the process of discovering truth from ancestral roots and the frequency of parent child interaction in investigating of disturbing conditions and matter. How both the ends respond i.e. positively or negatively? The truth model will also be assisting in understanding the scenarios of untruthfulness (social interaction) when opposite reactions insist students to act differently to prescribed criteria and standards. Various studies show that untruthfulness occur due to in hunger and starvation. Further, truthfulness is the adverse situation in anger or aggression (need to be checked). Some other factors such as social disorder, neglected by superior authorities (teachers or maestros) also result in provoking truthfulness in the students. The model will be quite helpful in checking of such disorders of truthfulness.
\end{abstract}

Keywords: Two-way Communication process, Disorders of truthfulness, Hunger and Starvation

DOI: $10.7176 / \mathrm{JESD} / 10-6-14$

Publication date:March $31^{\text {st }} 2019$

\section{Introduction}

Truth is always present and available into our surrounding, somewhere or somehow, it is in hidden or veiling state. Many thinkers have defined the truthfulness in many forms but their perspectives were common i.e. any state of feeling that reveal with reality of nature. The most thinkers view that we interact with the truth which in accordance to communication process - a single way process. But in reality, it is truth that also interacts with us at first. Thus, there is a two-way process of communication. Except, the truth is tried to be averted or dominated by the oppressors which in fact, the barrier, obstacle or hurdle in the between the communication of truth to four type of personalities. However, prevalence of truth is dependent on reliability of each personality type i.e. truth approaches, truth setter's, truth appointer's and truth outliers. Each personality has difference in approaching the truth and displays inner ability to explore and defend the truth from the oppressors (Sue, 2010).

The truth model discusses the two-way process of interaction (direct or indirect interaction). Each personality type is provided with a key word to recognize his / her role in the whole process. Whereas, the truth is also given with (Tr) symbol to understand its direction in communication. The oppressors has a very special role in seizing the way of communication with each personality but the personalities can also communicate with each other but in indirective manners. i.e. taking influence and inspiration from one's idea or conduct. There are many models that describe one to one relationship perceptive but they all responds in interpersonal models of communication. It is strived in this model, to inculcate the effects of extra personal communication mainly in the frame work but how interpersonal communication between groups more than one time is possible. This framework will also help in finding the right selection of this hypothesis (Feinberg, 2017).

Untruthfulness, is the adverse to truthfulness situation. According to humanitarian and social sciences studies, people told lie or intend to lie in extreme hunger and starvation and some of the thinkers suggests that they even lie in anger situation. Which in accordance to clerical thinkers is correct because they are at that situation under the influence of devilish grudge which intend them to lie for the sake of hiding their truthfulness (Robinson, 2018). The study also stresses upon prevalence and existence of truth within our surroundings, when oppressed by barriers. It also assists in finding out the situations that leads to provoking of truthfulness in a personality, most 
probably, the students in post-graduate and graduate standards dealing with unawareness, inherited qualities and social interaction (base hypothesis).

\section{Rationale of the Study}

1. Better understanding related to existence of truth around us.

2. Interaction of each elements of the framework with each other.

3. Clear and crystal understanding of extra-personal communication.

4. How the truth prevails in our surroundings.

5. The four type of personalities in the course of the truth communication. (interpersonal communication)

6. Impact of oppressors in the truth model. (role and existence of barriers in communication process)

\section{COMMUNICATION MODEL (TRUTH \& OPPRESSORS)}

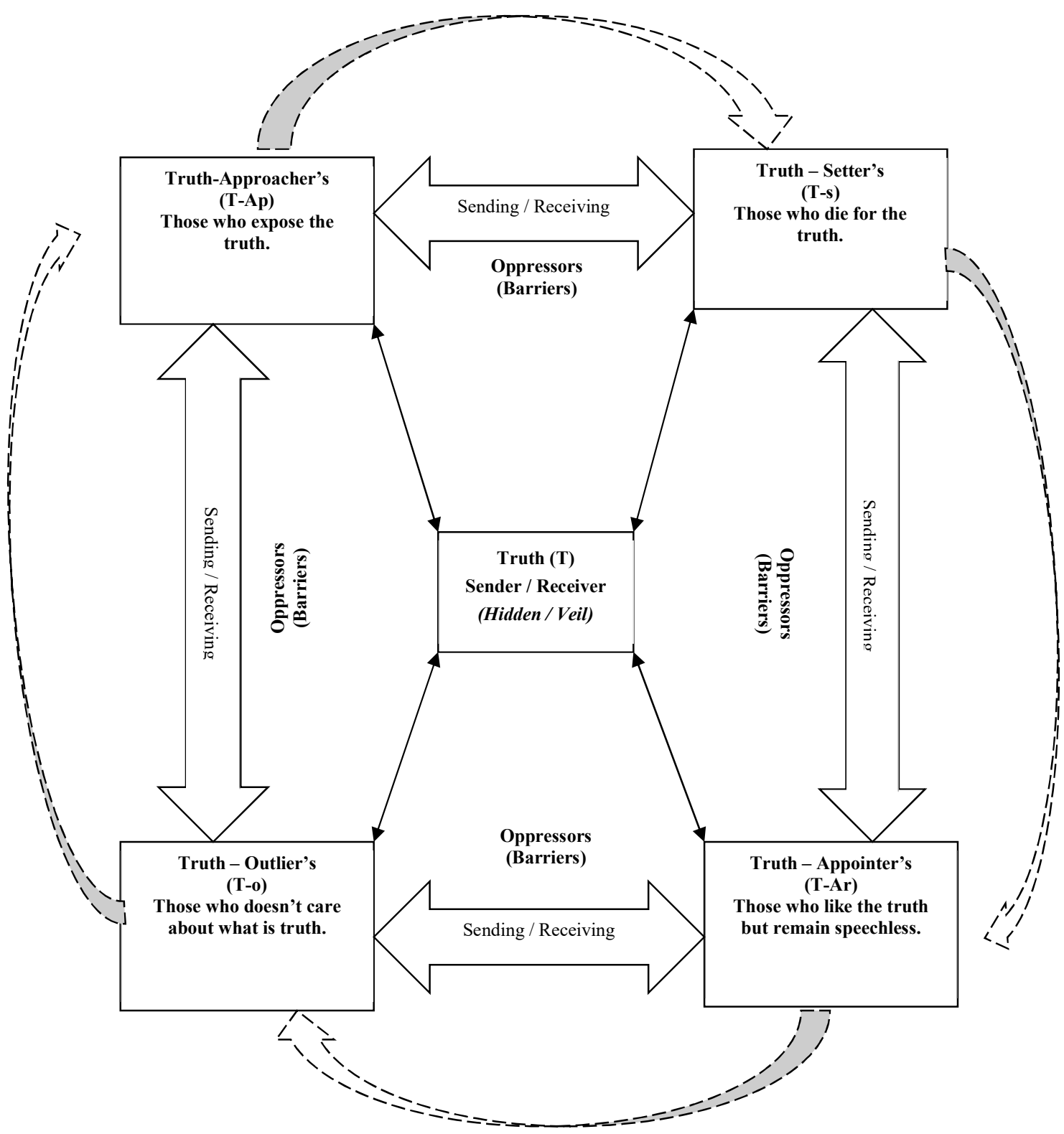

4 Type of People / Personality in Contrast to Truth

1. Truth-Approacher's (T-Ap)

Are those personalities who are always in search of exposing the truth but when oppressed or dominated by any behaviour during the process of communication they simply kept it with them. These people like to pace up with 
the truth but have no ability to hold with its after effects.

\section{Truth-Setter's (T-s)}

Are those personalities who died for the truth, no matter what the consequences may be, but they always keep themselves alive from inside at any cost i.e. positive or negative impact in the process. Thus, they have the ability to accept the truth and set an influence with their inspirational act.

\section{Truth-Appointer's (T-Ar)}

Are those who always there for the truth or in search of the truth i.e. like and accept the truth but they don't have much guts to respond in oppressive situations. They are weak from inside. However, they always kept the truth with them for better or favourable situation.

\section{Truth-Outlier's (T-o)}

Are those personalities who does not care about the truth. They don't even have boil up guts to stand for truth. They might also be opportunist's i.e. cope up with any kind of situation.

\section{Oppressor's (Barriers)}

Are those media which become barriers, hurdles or obstacles in the way of these communication factors. They are everywhere, in any form i.e. physical interruptions, noise, voice, social and political conflicts and media of transmittal etc. Their utmost motive is to block or undo the communication between truth and the four elements. They might become dominating in playing their role but-

The truth naturally prevails through any four of the elements and remain, sometimes under disguise, thus, it is a two-way process i.e. direct or indirect but oppressor's plays a direct intervention role.

\section{Truth (Tr)}

It is the centerfold of the model and played two way process i.e. sending and receiving of message and communicating to any one of the four elements in the framework (Kani, 2002).

\section{Research Findings}

The study conducted through questionnaire and found results from statistical measures open a new dimension in understanding the truthfulness and untruthfulness in a personality. The study also assists in finding the results of the hypothesis in favour that somehow, truthfulness in a person depend upon the three essential factors i.e. truth traits, truth inheritance and truth interaction in social circle; that lead him / her to uncover the truth from his / her surroundings. The study was conducted with 200 respondents, all from various study levels of university of Karachi i.e. undergraduate to graduate and semester and annual exam criteria. The findings suggested that:

1. Majority of respondents have a behavior of truth setter's (stand and stay with the truth and trend it). Some of the respondents exhibits the ability of truth approacher's i.e. exposing the truth. But unanimously, the result shows the inclination as accepting the truth and discovering for new dimensions i.e. analytical approach of studying.

2. The traits also exhibit the adaptation of the view that truth also communicate with us either openly or in veiled form, which suggests that the nature of communication and the four elements is extra personal and as well interpersonal at the same time.

3. The age of truth uncovering or knowing about the truth is unanimously found at the adulthood which suggests the behavior of understanding the truth at the maturity and practical age. This is where, they also started to imply it with practical implications.

4. The sources of uncovering the truth were majorly found from religious clerics / maestro's and self-study. Which suggest another dimension of believing on the factual knowledge on the basis of cross check reference trait.

5. The result suggests that most of the respondents have a prior knowledge about their ancestral roots of knowledge which also intend them to realize the truth and start digging up about its various dimension of truth.

6. However, the results of parental integration suggest the slight weak relationship between parent and child coordination which might be the factor responsible for declining the inclination trait of truth exploration.

7. The results suggest that there is averagely questioning in the parental integration which is also the factor of staying not updated with the real facts.

8. The learning beliefs on the other hand are satisfactorily found from the studies, this indicate the source of trust and integrity with the source of exploration.

9. The results suggest that most of the students have a habit of hiding the truth based upon the habit of intended lie from their parental sources and also suggests that encouragement and discouragement from parental source is very weak in nature.

10. Poor social circle interaction is also the essential factor in discovering about the new knowledge and facts Which also affect the encouragement / discouragement sentiment of the students adversely.

11. Majority of observing responses suggest that when a disturbing situation is acquired with proper knowledge from Maestro's for an encouragement and discouragement. The reaction is satisfactorily in favour and this lead the students to stay with the truth or facts that are discovered. 
12. Cross reference providence indicators and cross check ability for its reliability / vulnerability suggest that the poor interaction results adversely on truth exploring ability and might lead to dissatisfaction on staying up with the facts.

The results of the study are quite helpful in suggesting that truthfulness is significantly dependent on three major factors discussed in hypothesis and will be helpful in strengthening the ability of truthfulness. If they are not found in order and discipline untruthfulness will be the adverse situation.

Further, oppressors have a dominant role in weakening the truthfulness ability in the student's behavior but it can be minimized with several measuring units however, cannot be eliminated from the communication process.

People are often found with the intention of uncovering the truth and the prevalence indicator is essential in playing a positive role in discovering about the truth.

Hypotheses

1. Truthfulness or untruthfulness is due to cultural inheritances.

(Dependent Variable $=$ Truthfulness $/$ Untruthfulness - Independent Variable $=$ Cultural Inheritances)

2. Truthfulness or untruthfulness is due to social integration.

$($ Dependent Variable $=$ Truthfulness $/$ Untruthfulness - Independent Variable $=$ Social Integration $)$

\section{Research Queries:}

1. Why barriers always have direct interruption role?

2. Can they be minimized, meanwhile in dominating stage?

3. Why people need to interact with the truth?

4. Why the truthfulness / untruthfulness dependent on prevalence of truth?

Research Method

A qualitative research with 200 sample size as response to inference the measures of base hypothesis. Keeping in use of semi structured data questionnaire containing open and close ended questionnaire revealing survey interview with well-versed respondents as a primary source of data collection. The core check on hypothesis shall be done through research material collected from various online and offline books and as well internet articles, journals etc. Relevantly explaining the insight for data measures as a secondary source of data collection.

\section{RELEVANT LITERATURE REVIEW}

People often cheat or lie when they are favoured or encouraged about it whereas, truth is just an adverse of it. However, some psychologist suggested that people often lie in the state of extreme hunger, poverty, tiredness and when they are exhausted in their life (Ariely, 2013).

This is where dishonesty starts, it is when we get tired to resisting temptations of ours and this where we started to cheat or lie with others even though with ourselves sometimes.

\section{Honesty and Dishonesty}

"Honesty is the best policy" (a famous quote) to influence people towards telling the truth. In other quote also truth is not easy to tell in every situation i.e. "It is better to lose by telling a truth rather gaining triumph on the burden of lots of lie". (Islamic Cleric - Hazrat Ali A.S). The said quote is very important to understand the significance and roots of telling the truth under different situations i.e. oppressed or favoured.

People often sought to tell a lie under pressure (oppression) if their mind is sided with truthfulness, vice versa would be the situation of favorableness. According to re-known authors and scholars and psychiatrists; when telling a truth, naturally some core problems that may arise in the course (Boyer \& Pascal, 1994) :

1. People admit the truth of a sentence except for its paraphrase. They judged it by certain utterances - problem of 'Literalism'. i.e. truth is expressed but not conveyed.

2. Sometime, context is left aside in contrast of utterance - problem of 'Linguistic-ism'. i.e. truth expressed in other languages.

3. When people admit truth as true but never behave in accordance to its conviction - problem of 'ill-convictism'

4. People seem to have truth in many counters - intuitive statements which conflict with what they admit in other context - problem of 'Ignorance'.

When in any criteria, if the following problem arise during the course of truthfulness these four elements or behave has the boundary to measure honesty and dishonesty in a person. Meanwhile, it might also be the resultant of simply barriers in communication (Sue, 2010).

\section{The 4C's of Truth in Communication}

"Truth is Truth - to the end of reckoning"-William Shakespeare (Measure for Measure, Act V, Scene I).

1. Comprehension - People admitting the point of discussion but not believing in the source.

2. Connection - people are getting the message but not connecting personally etc. either with it.

3. Credibility - when message has its weight but the source isn't.

4. Contagiousness - when everything is working but no excitement at all to the receptor side (Albanese, 2007) . The fact of the matter is how the barriers arise in the course of communicating the truth in the surrounding. We 
must first discuss the main barriers to understand such dilemma:

\section{Common Barriers to Effective Communication}

1. Emotional, or Taboo - most disturbing topics in the society that everyone feel guilty of not discussing them in public.

2. Difference in perception - the two distinctive ideas or point of thinking.

3. Physical barriers to non-verbal communication.

4. Expectations and Prejudices - false assumptions.

5. Role - Interaction between superior to inferior relations.

6. Biasness - preconceived beliefs for others.

7. Misinterpretations - jumping to conclusions very early in the course of communication (www.skillsyouneed.com/ips/barriers-communication.html)

Thus, barriers play a vital role in the course of truth but most of the school of same thoughts discuss it from other point of view that sometimes it is barred not from outside barriers but it is our inside 15. People are often caught telling a lie for passion or they might be under some pressure but most of the time they are willing to do it of their own will (Patton, 2017). Some theorists suggested some signs of truthfulness, untruthfulness and the different type of lies:

\section{Signs of Truthfulness}

The following signs are the precursors to determine the truthfulness in someone accent or views:

1. Longer or detailed story - people often when tell a truth they present it in detailed and summarize style.

2. Right Amount of Eye Contact - face to face communication.

3. Steady Breathing - face to face, interpersonal communication.

4. Steady or undisturbed voice.

5. No Blaming Game for others.

6. No Nose Touching - in dis-contention people often do such mistakes.

7. No Throat Covering - in dis-contention people often do such mistakes.

8. No change in rate of blinking of eyes.

9. Complete syntax of sentences - use of proper grammar.

10. No fake smile.

11. We believe on them by our own instincts.

12. Answering questions with questions - untruthfulness.

13. Taking oaths unusually - untruthfulness

14. Lack in self reference - untruthfulness.

15. Equivocation - exemplifying each statement.

16. Ambiguity - feeling depressed or despair in between the talking.

Different Type of Lies

"I swear to tell the truth" - Lies of commission, an untrue belief of being truth.

1. "The whole truth" - Lies of Omission, a lie that contains an important part of information.

2. "And nothing but the truth" - Lies of Influence / Character, willingly doing it (Advance) .

Conclusion

"Truth is truth, if no one is doing it, Wrong is wrong, if everyone is doing it." (a famous English quote)

The communication between the truth and its elements is always a two-way process but due to intervention of barriers (oppressors) its impact remains weak and dull for the time. However, the truth always revealed from various channels. The impact of oppressors on truthfulness of a person remain constant until he / she intendedly show his / her inclination towards knowing about the facts and its discovery. The signs of truthfulness are very helpful in placing a person's sentiment as a fact or fake, interpersonal relationship are playing pivotal role, too. The major barrier in the path of truthfulness is the person's own integrity. If it is at the positive and higher-level truthfulness is ensured if not, the vice versa will be an output. However, physical barriers can be minimized with self-determined and system integrated approach. People often tell a lie when they are in extreme hunger situation (materialistic or non-materialistic) in order to fulfill their needs.

\section{References}

Retrieved from www.skillsyouneed.com/ips/barriers-communication.html.

Advance, M. (n.d.). Retrieved from Michiel Advance - Twitter.com Netherlands

Albanese, I. (2007). The 4C's of Truth in Communication: How to identify, Discuss, Evaluate \& Present StandOut, Effective Communication. Paramount Market Publishing.

Ariely, D. (2013). The Honest Truth About Dishonesty: How We Lie to Everyone--Especially Ourselves. Harper Collin Publishers.

Boyer, P., \& Pascal, B. (1994). Tradition as Truth and Communication, A Cognitive Description of Traditional Discourse. Cambridge University Press. 
Feinberg, M. (2017). Curtain of Lies: The Battle Over the Truth in Stalinist Eastern Europe. Oxford University Press.

Kani, J. (2002). Nothing But the Truth. Witwatersrand University Press.

Patton, M. Q. (2017). Principles-Focused Evaluation: The GUIDE. Guilford Publications.

Robinson, N. S. (2018). A Sojourner's Truth: Choosing Freedom and Courage in a Divided World. InterVarsity Press.

Sue, D. W. (2010). Microaggressions in Everyday Life: Race, Gender, and Sexual Orientation. John Wiley \& Sons.

www.bustle.com. (n.d.). Retrieved from www.bustle.com/articles/165346-11-way-to-tell-if-someone-is-tellingyou-truth-according-to-science. 